\title{
Improving Dissemination and Localization of Cultural Heritage Through Multimedia Maps - The Case of Lipari Island
}

\author{
Nicola Maiellaro ${ }^{(凶)}$, Antonella Lerario, and Antonietta Varasano \\ Construction Technologies Institute, National Research Council of Italy, \\ via Lembo 38/B, 70124 Bari, Italy \\ \{maiellaro, lerario,varasano\}@itc.cnr.it
}

\begin{abstract}
Most of the maps in tourist information websites show only the position of the Points of Interest (PoIs) - providing sometime a link to a webpage - making choices difficult. Multimedia maps, instead, could support users in satisfying the traveler needs giving links to information about the PoIs. The developed application supports improved connections between documents and the places they refer to because the user could select PoIs to visit through previews of its multimedia documents. PoIs could also be filtered through their categories and types, accessibility status and time line, thus improving the system usability. This article describes a multimedia map developed as sample for the Lipari Island, the largest of the Aeolian Islands (in Sicily), inscribed in the UNESCO World Heritage List.
\end{abstract}

Keywords: Multimedia map $\cdot$ UNESCO $\cdot$ RESTful web service GeoJSON

\section{Introduction}

The World Economic Forum identified [1] two main categories of users: 'over 60' and 'Millennials'. The former practice non-seasonal tourism, requiring accessible services and high quality standards; the latter are particularly interested in cultural experiences even in less known destinations, regardless of the level of services. Moreover, $95 \%$ of travelers use digital resources not only to book but also to search for information, plan the trip and share their experiences [2].

Although there are many websites containing a great amount of information, it is not easy to find proper and tailored information satisfying various traveler needs, due to a poor attention to the potentiality of interactive maps gathering and sharing multimedia information. Most of the maps in tourist information portals show only the position of the Points of Interest (P.o.I.s), sometime with a link to a webpage.

Multimedia maps, born with the development of the personal computer since the 1980s [3], interact with multimedia documents through mouse clicks on hypertext links available in 'hot-spots' [4]. Another way, providing descriptions details (in hypertext format), photo, and video-organized in three tabs in the callout-has been suggested in [5] but now not available online [6]. 
In our novel approach, multimedia documents and P.o.I.s are linked in bidirectional way: the user could start selecting a P.o.I. in the map and access its multimedia data through their previews (in order to select the objects to visit), or viewing the multimedia documents available in the map and select the owner P.o.I. to visit.

In order to reduce the great deal of time and efforts to produce this kind of mapsunfamiliar to many - an authoring system has been developed, using two sets of files with info about P.o.I.s and the related multimedia documents.

\section{The Case Study}

Lipari, the largest of the Aeolian Islands in Sicily, is an outstanding testimony of the processes of generation and destruction of islands due to volcanic phenomena, still underway in other islands of the archipelago such as Stromboli and Vulcano. The whole archipelago was inscribed in the UNESCO World Heritage List in 2000, as remarkable example of the coexistence of two types of eruption (Vulcan and Strombolian), and can be considered as a constantly evolving archaeological park, enriched with naturalistic areas. 'Wild' areas can hardly be found in the whole Aeolian archipelago; the actual landscape is the result of a close intertwining of man and nature, of the natural environment and a millenary human work [7]. The succession and overlapping of anthropic activities over time ended up marking this territory in negative sense, starting from the chaotic building development in the '60s up to the growth of tourism-related activities, especially in coastal areas, with often-irreversible effects leading to a differentiation among the Aeolian Islands. Lipari and Vulcano can be considered as being in their mature stage as tourist destination; Lipari, in particular, though having a weaker volcanic component, has undergone mass tourism more than the other islands thanks to its closer connection with the rest of Sicily and to the higher level of its accommodation facilities. The latter, anyway, faced with the recent explosion of over-tourism in the summer, is now struggling to bear such burden in a context where general services turn out inadequate even for residents [7].

Tourism, precious in the critical post-war period, is by now one of the two main causes for the environmental deterioration of the whole archipelago, and of Lipari in particular, due to its unrestrained and disharmonic development. The other cause is to be found in the current climate changes, in relation to the extreme vulnerability of coasts, determining a sensible and progressive rise in the relative sea level and the subsequent gradual submergence or flooding of valuable archaeological and cultural sites along the coasts, such as the port and the Church of 'Anime del Purgatorio'.

In this respect, the flooding scenarios outlined up to 2100 [8] are undoubtedly alarming. Such situation suggests the opportunity to protect this endangered material heritage from within an organic view, such as UNESCO can ensure. The designation in the WHL can potentially prove decisive also in relation to economic development, through tourism-related activities: the current local offer is still focused on the conventional 'sun-sea-and-sand' segment and on a young target, and it is not fitted for a demand referred to the volcanic area [9]. However, although recognizing the extreme fragility of coastal zones, new promotion strategies still head towards them, by 
proposing thematic or geo-tourist itineraries [10], without sensibly impacting in terms of flow de-seasoning, which is instead more and more necessary [7, 9].

Much research work has been carried out, in general, on the effects of WHL designation on heritage management and on territorial development [11-16]. Among them, and with specific reference to tourism, some are of the view that the UNESCO 'brand' in itself can have only indirect impacts and that the inscription in the List is not sufficient, alone, to exert long-lasting effects [9].

On the basis of the 'public good' nature of UNESCO heritage and of the analysis of the performance observed in the Aeolian Islands [17], it derives that the main determiner for a correct territorial development is the whole of virtuous processes that are concretely triggered upstream and downstream of the designation, i.e. preparatory and subsequent to it, bringing about the production of further public goods [18]. More specifically, the effectiveness of the UNESCO recognition is linked to the site's ability to activate creative processes in different sectors.

Creativity is, then, an essential factor for the positive development of a UNESCO site. Actually, this is an element that marks the evolution of WHL recognition criteria, gradually shifted along the years from historic and artistic value to the consideration of immaterial culture, up to the current focus on the relation between heritage and local community and on creativity.

In our vision, knowledge plays a key-role in this virtuous relationships between material (physical resources) and intangible (the ways communities relate to them) culture, even more for a threatened heritage as is the case with the Aeolian Islands. Alongside with heritage, also knowledge on it requires careful protection and promotion, and the tools designed to support it can effectively generate creative processes.

Indeed, creativity can be expressed also in the ways residents and visitors define and plan their visit experiences based on knowledge available. More specifically, tools conceived for interactive use facilitate, on one hand, knowledge acquisition, concretely realizing one of the basic principle of the current UNESCO perspective: the 'transmission' - especially in intergenerational sense [7] -, of heritage. On the other hand, interactive tools, enriched with multimedia content, support creativity in the establishment of relationships between users and resources, by allowing personal approaches in the planning of visits, the selection of resources, the definition of physical or thematic paths combining them and, ultimately, in the logics of appropriation of their meanings.

Furthermore, giving more visibility to unknown inland resources alongside with coastal sites through the use of tools able to connect - though not physically - heritage and people, also lets unexplored resources come to light, rebalancing and rewriting the overall predominant image of a region.

Within the framework of the project 'Mu.S.A.-Must See Advisor', we have developed multimedia demonstration maps on the cultural heritage of Matera and Lipari, included in 'Mirabilia -European Network of UNESCO Sites' project managed by the Chamber of Commerce of Matera. The project started in 2012 as a joined initiative of thirteen Chambers of Commerce in Italy; it is committed to the promotion of unexpected places of historical, cultural, and environmental importance in the UNESCO World Heritage Sites [19]. 
Currently the map of the general catalogue of cultural assets developed by the Ministry of Heritage and Cultural Activities and Tourism of Italy [20] does not contain any point of interest in Lipari (Fig. 1).

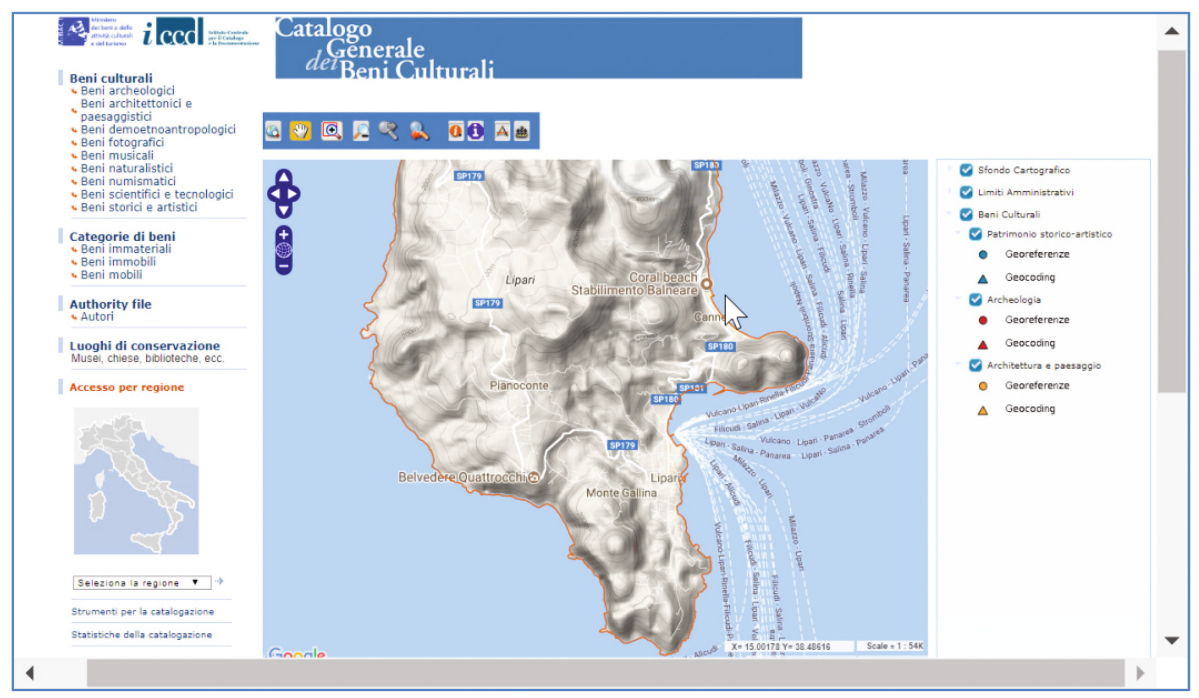

Fig. 1. General catalogue of cultural assets - Lipari Island [20]

The map of Parks, Museums, Galleries and Archaeological Areas developed by the Department of Cultural Heritage and Sicilian Identity [21] shows a single point of interest: the Regional Archaeological Museum 'Bernabò Brea' (Fig. 2).

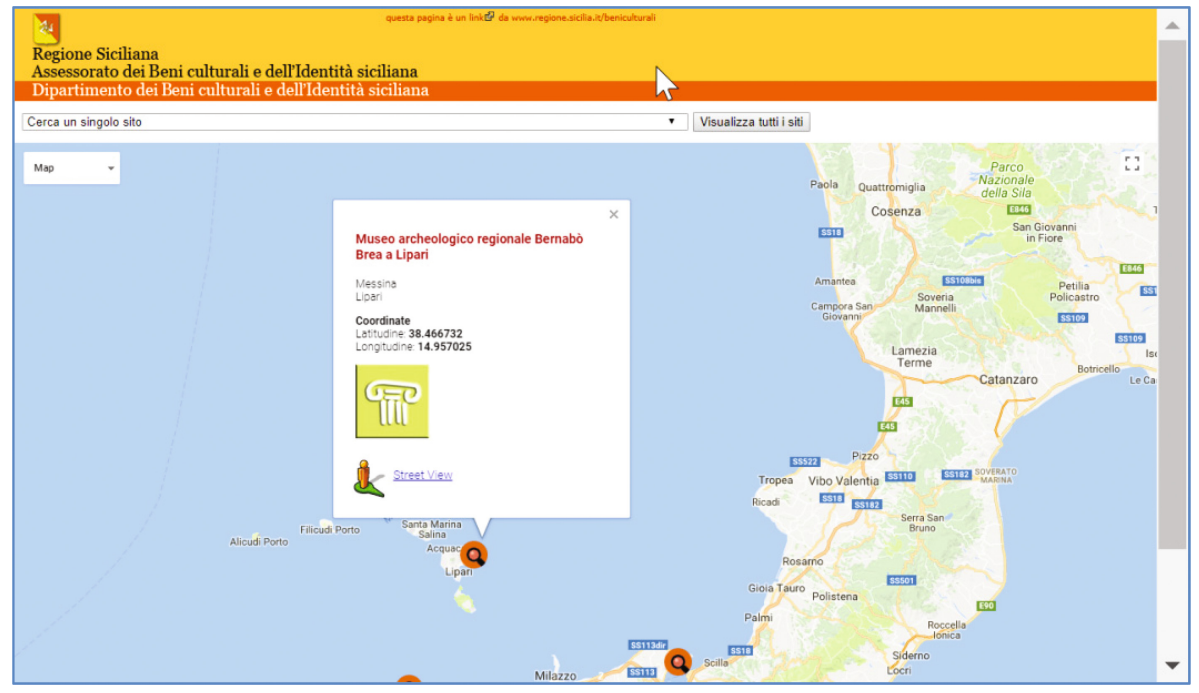

Fig. 2. Map of Parks, Museums, Galleries and Archaeological Areas in Lipari Island [21] 
The official website of Lipari [22] has a static map with the following P.o.I.s, described in different webpages: the Castle of Lipari, the Cathedral, the Norman Cloister, the Aeolian Archaeological Museum and the Diana's Park (Fig. 3).

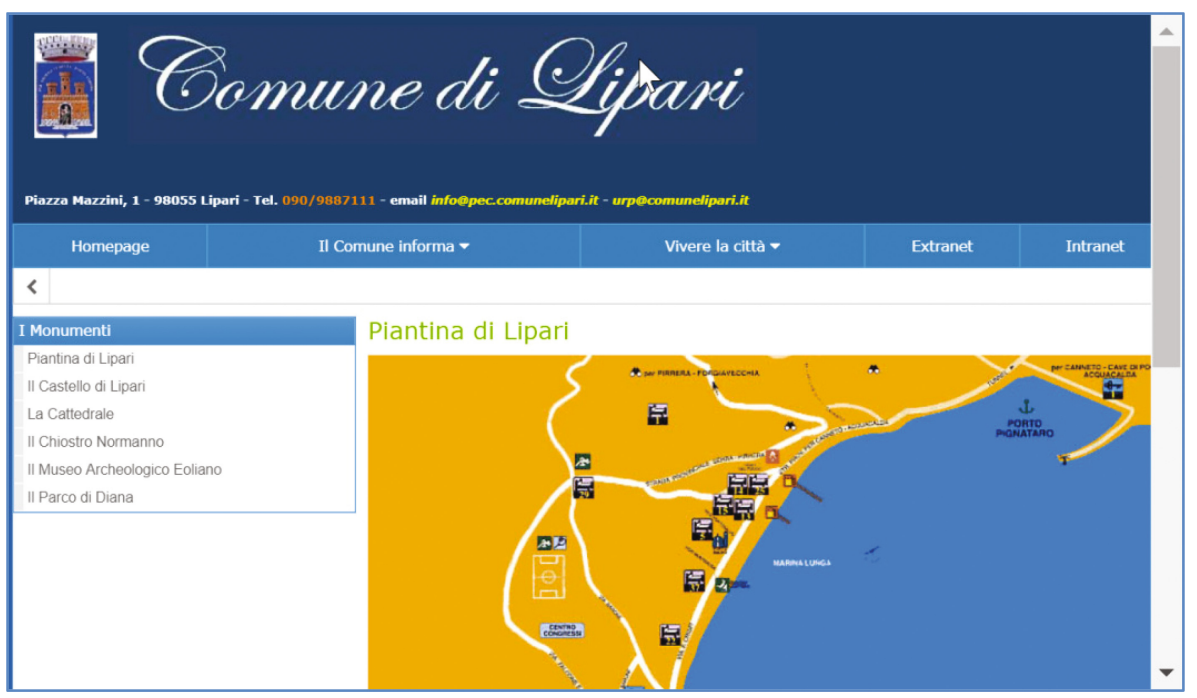

Fig. 3. Monuments in Lipari Island [22]

In the maps there is therefore very little information on P.o.I.s in Lipari, although there are 44 archaeological areas listed in the 'Aeolian Islands UNESCO Management Plan' [23] localized in detail scale (Fig. 4) and in a map in 1:10000 scale (Fig. 5).

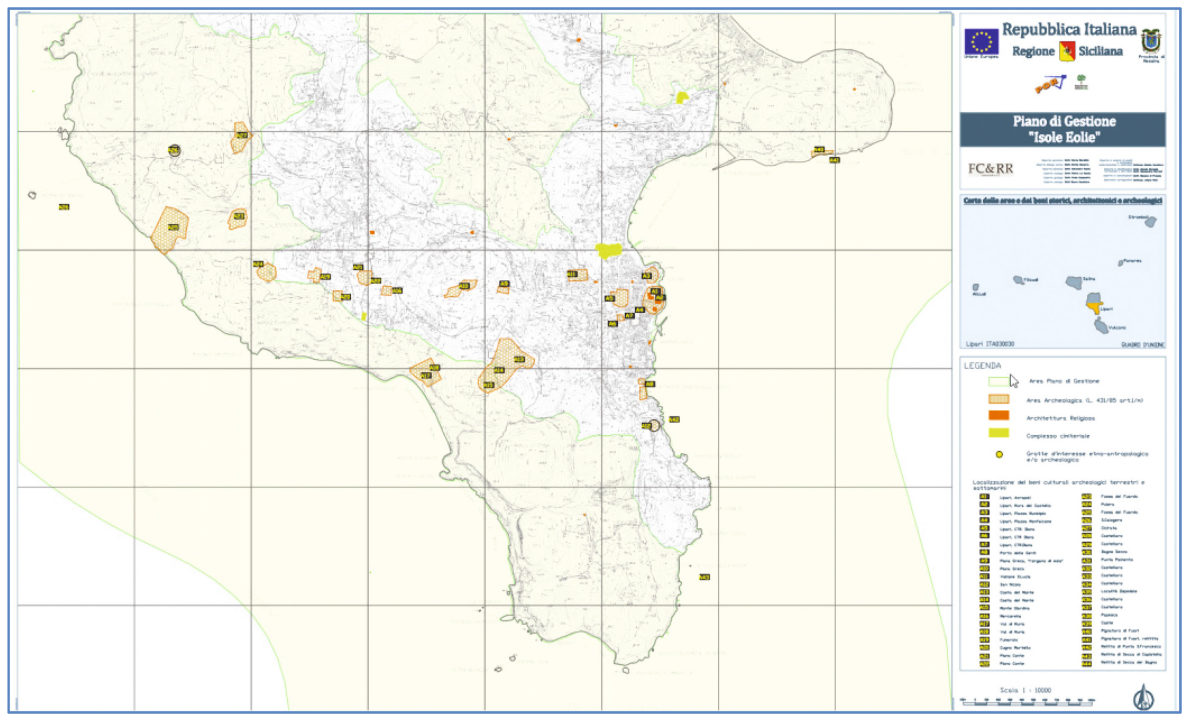

Fig. 4. Map of the historical, architectural and archaeological areas and assets of the 'Aeolian Islands' Management Plan 


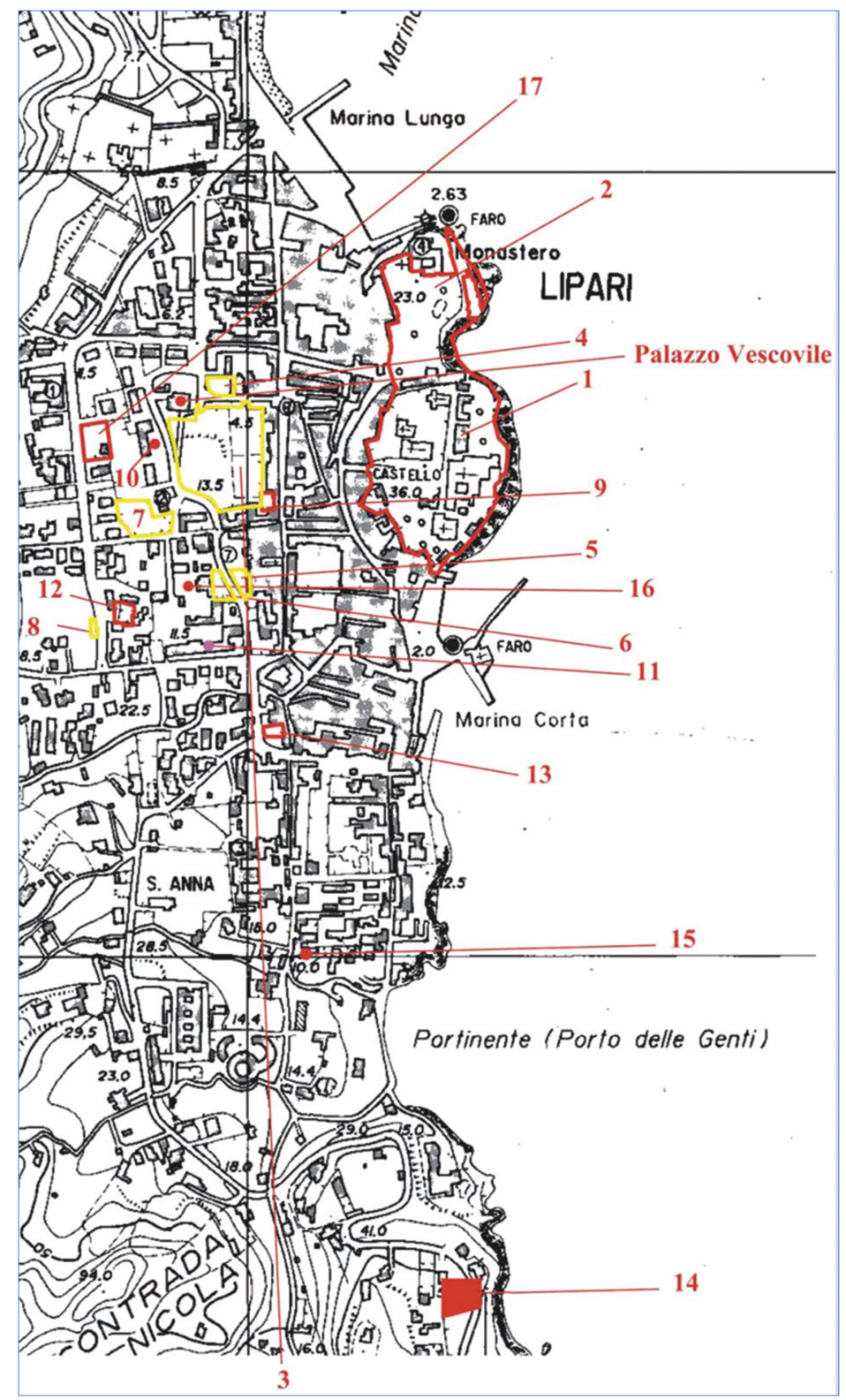

Fig. 5. Aeolian Islands UNESCO Management Plan, Cartographic attachments [23]

Moreover there are a couple of road signs (Fig. 6) holding info about historical and archaeological sites of Lipari Island.

This short overview shows the need to attract the attention of institutions towards cultural heritage promotion through new communication tools that can improve the visit experience. To this end, using only information available on-line, a multimedia 


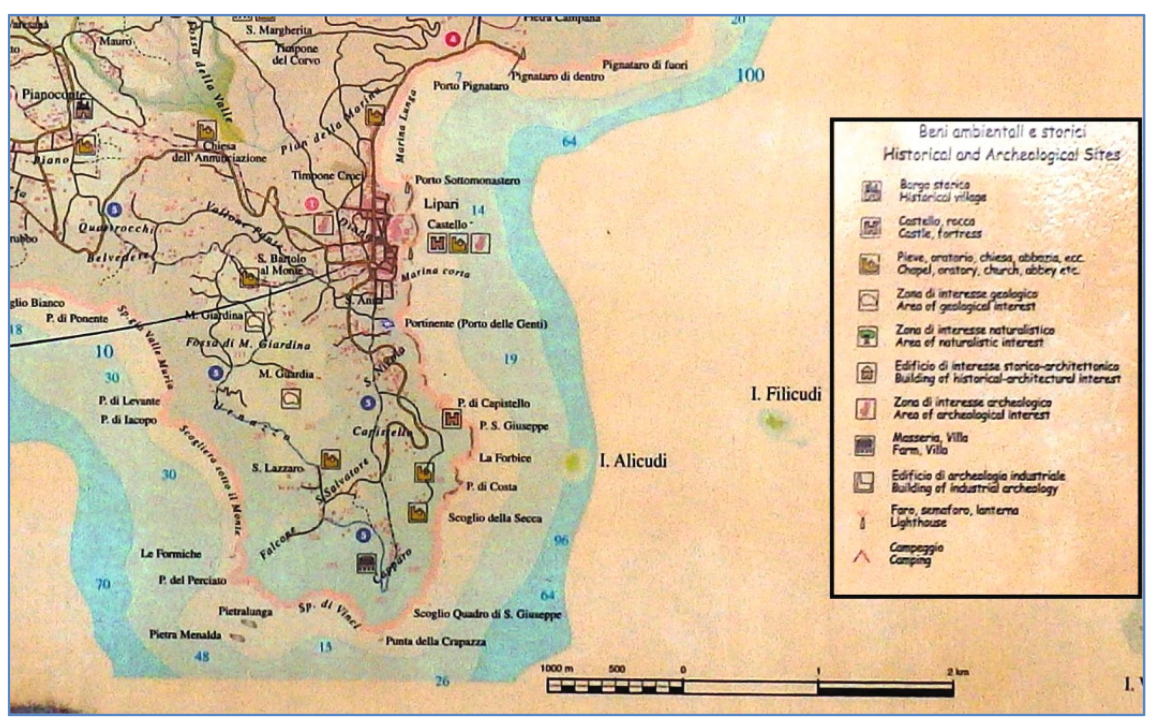

Fig. 6. Road sign (detail): Lipari Island-Historical and Archeological Sites

map of natural and cultural P.o.I.s of Lipari Island has been developed (Fig. 7). In order to give an example of the interactive map potential, the P.o.I.s have been grouped in five Categories and, for the case of the category 'Archaeologic site', in four Types.

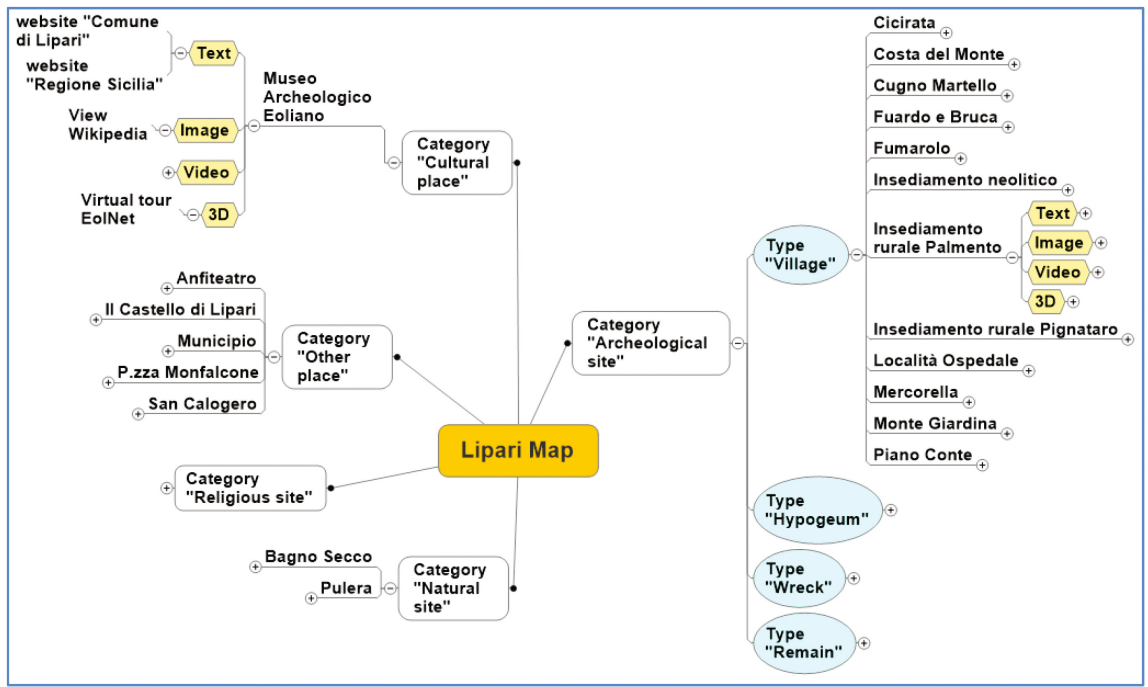

Fig. 7. Lipari Map overview: P.o.I.s grouped in Category (in transparent rectangular topic) and Type (in light blue oval topic); each P.o.I. has its own documents (in yellow hexagonal topic), grouped in Text, Image, Video, and 3D. (Color figure online) 


\section{The Map Generation}

\subsection{The Authoring System}

In the last few years, cloud-based services have been playing an important role in largescale web-based applications.

The authoring system developed (Fig. 8) is a practical and exhaustive example of cloud-based service for data management and integration.

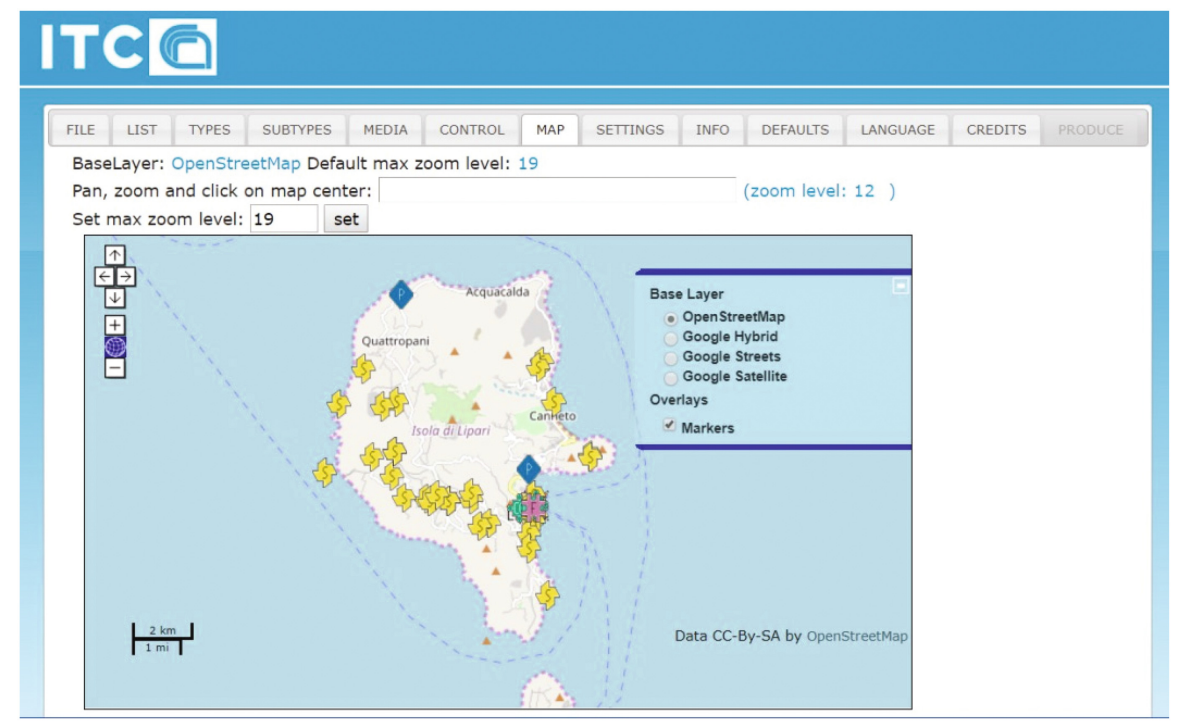

Fig. 8. The authoring system for multimedia map production: tab 'map' for preview/settings.

This system allows developers to import local data from spreadsheets file format into cloud-based services. As result, cloud data can be easily integrated to own website. Indeed, the authoring system enables users to upload tabular data files in the 'DBF' format, even if it is possible easily to extend the ability of system upload functions to import extra file types, such as CSV and spreadsheets.

Automatically and during end-user upload procedure, the authoring system supports the integration of data by performing joins across tables that may also contain different data stored in discrete files.

At present, the data have been stored in two files:

- a spatial set (P.o.I.s List file), including latitude and longitude of each P.o.I.;

- a document set (Media List file), including objects (grouped by text, image, video, and 3D content) contained in P.o.I.s.

This system has the ability to aggregate the data and provides multiple ways of visualizing the data using a map and a HTML table (DataTable). 
The map provides geospatial visualization of information by create a marker on each location/P.o.I. so that users can analyze and understand the relationship between data and geographic location. The HTML table provides an immediate option to visualize, modify and analyze data.

The storage aggregate automatism, carried out in accordance with the entityrelationship (E-R) model, builds and verifies the entities or 'items' of an aggregate [24], making a JSON data store. The E-R model of the authoring system is the result of systematic analysis in recent years. We applied E-R principles to the physical modeling of JSON data store, which is non-relational in nature and its relationships are implicit.

Entities are characterized not only by implicit relationships, but also by additional properties (attributes) and identifiers (primary keys) need to aggregate data.

An aggregate consists of four entities (see Fig. 7): Category, Type, P.o.I., Media Object (text, image, video, and 3D). It compares the data in the P.o.I. List file with the Media List file to ensure that the interconnectedness of entities, relationships and their attributes are completely aligned, synchronized and meshed together with their cardinality [25].

All functions for serving as user interaction purposes are implemented in JQuery, where visualizations are interactive and dynamic based upon user's requests and DataTable jQuery plugin [26], integrated into the system, is a perfect choice in order to accomplish this task.

DataTable supports client side searching, sorting, pagination (client/server side) through ajax/html request and exposes a lot of functionalities for server side processing, such as any list data (table) can be converted to JSON format, without any issues, and send it as Ajax response.

Using the command 'Produce' in the authoring system, the end-user can quickly and easily create an attractively formatted web page, even if he is not familiar with HTML coding. After creating a web page and producing a multimedia map as a 'Single-Page Application' (SPA), which is a different way of building HTML5 applications from traditional web page development [27], the end-user can post it to an Internet or intranet location.

A SPA is one web page that works inside a browser and does not require page reloading when the end-user interacts with the app, so that JSON data are combined with reusable template to create views that make up the SPA's User Interface (UI) [28].

Instead of spreading the functionality of the multimedia interactive map across a collection of separate web pages with hyperlinks among them, a single root page has been defined: the application uses the JSON data to update the page dynamically, without reloading it and separates the UI library and the data.

\subsection{The Map Architecture}

While the potential of SPA is promising, implementing them can actually be tricky due to search engine optimization (SEO). Search engines are still the most popular way to discover new products and find information. The SPA interactive map (see http://www. itc.cnr.it/ba/map/LPR/) runs AJAX [29] calls and client-side scripts (Javascript) while search engines do not run scripts within pages, so, today SPA-based map is not completely indexed by search engine crawlers and it is necessary that all AJAX 
requests in web application can also be requested by static links [30]. Google is claiming to be able to index website content generated using JavaScript [31] and saying it will crawl and render AJAX-based websites as they are [32].

Today, effective ways to find and access existing datasets distributed across a range of repositories are increasing requested. Therefore, our research is focusing on improving the discoverability of data; part of this will involve using information about how data is searched for (search terms, location, etc.) via web application and making data easier to interpret and use across different formats; visualizations and APIs will be also key to maximizing its beneficial use and impacts.

Over the past few years, single-page web applications and their frameworks have gained huge popularity. The SPA solution has been adopted for its main advantage of improving the web surfing of the multimedia interactive map on mobile; it is able to:

- Affect the positioning and the discoverability of data on the web, since the usability on mobile devices is now a ranking factor.

- Support rich client-side functionality that does not require reloading the page as users take actions or navigate between areas of the app.

- Load more quickly, fetching data in the background, and individual user actions are more responsive since full-page reloads are rare.

- Run in a disconnected mode, making updates to a client-side model that are eventually synchronized back to the server once a connection is re-established.

- Concentrate all content in a single page, which is useful when we are targeting very precise queries.

The SPA solution is fast and user-friendly; it supports RESTful APIs and enables the distribution of the processing workload between the server and client computers. Finally, it is much easier to convert such web application into a mobile one.

The multimedia map is built on client-side JavaScript web application framework based on the model-view-controller (MVC) software architectural pattern and utilizing server side RESTful web services to provide desktop-like rich browser interfaces.

This architecture is designed on the service-orientation design principles of reusability, simplicity, extensibility, thus causing a clear separation of component responsibilities compare to 'traditional web application architecture'. Ajax and RESTful Web services naturally fit with each other and leverage widely available web technologies and standards, such as: HTML, JavaScript and XML/JSON [33]. For this system, there is absolutely no need to install or configure any other component to enable effective interaction between Ajax front ends and RESTful Web services. In fact, RESTful Web services provide Ajax with an application-programming interface (API) to deal with the interactions with resources on the server. Furthermore, Google Maps API is integrated in the application for rendering geospatial data within a web browser and for accessing rich mapping features and it is 'optimized' for smartphones with a set of APIs developed by Google, which allow communication with Google Services and their integration to numerous other services. 


\section{The Map Interface}

The desktop map interface has three main components: 'Menu', 'Callout' and 'Sidebar', which are closed in the default status (Fig. 9) to maximize the map, and opened on user action (Fig. 10). P.o.I.s are visualized on the map through markers with unambiguous colors [34], with different shapes and image according to category/type.

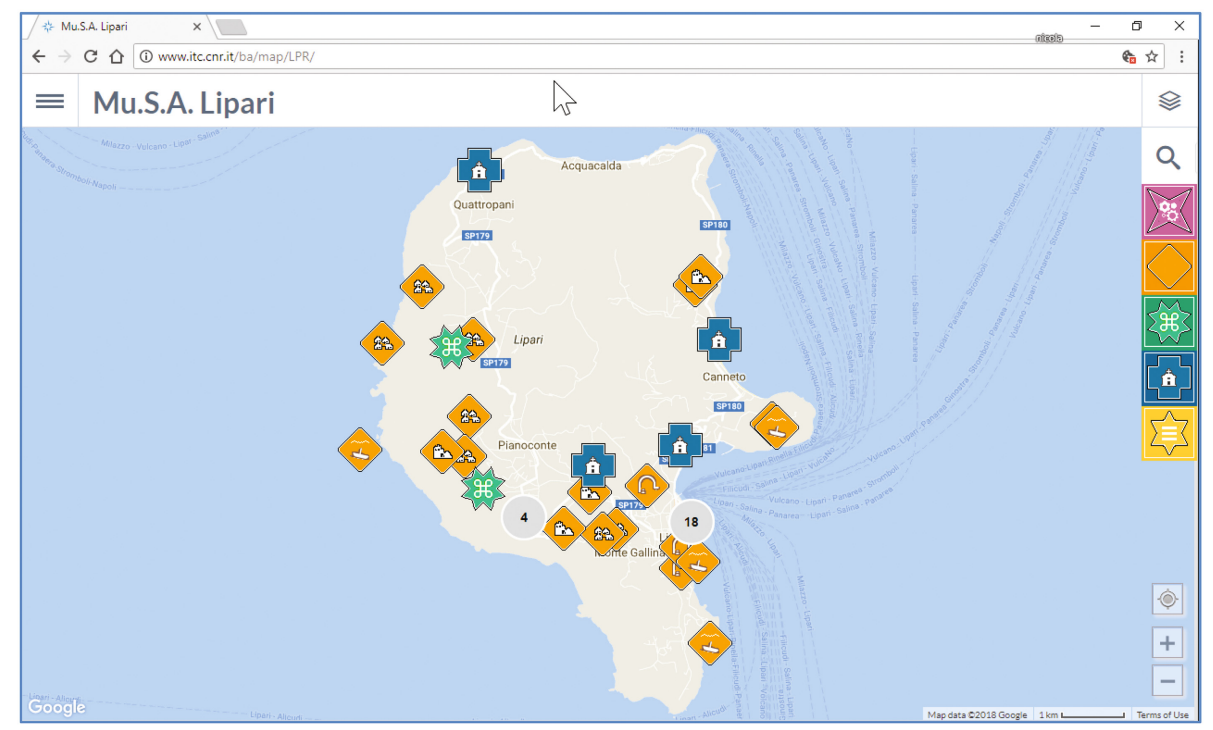

Fig. 9. Desktop map interface of Lipari island (http://www.itc.cnr.it/ba/map/LPR). It has three main components ('Men', 'Callout', and 'Sidebar'), which are closed in the default status. P.o.I.s are visualized on the map through markers with unambiguous colors. (Color figure online)

Menu. This component opens a list of functions applied to the map in two ways. With mouse over the 'Menu' symbol, the list displays only 'self-describing' symbol; clicking on the 'Menu' symbol, the list shows/hides symbols and functions names (Fig. 10, left):

- 'Best site', locating on the map the most interesting site.

- 'Satellite', switching on/off the map to earth view.

- 'Slideshow', opening a window with a moving set of thumbnails (characteristic square image of each P.o.I., used also in the callout and in the sidebar).

Clicking on a thumbnail, the map centers itself on the P.o.I. which the image belongs, opening its callout-this function is useful for totem installation because attracts the public attention.

- 'Time slider', updating geographic distribution of markers according to the construction century selected by the user in the timeline graphic through a handle control.

'Folder', displaying the preview of multimedia items (belonging to all the P.o.I.s in the project) item by item in a dynamic resizing window. 


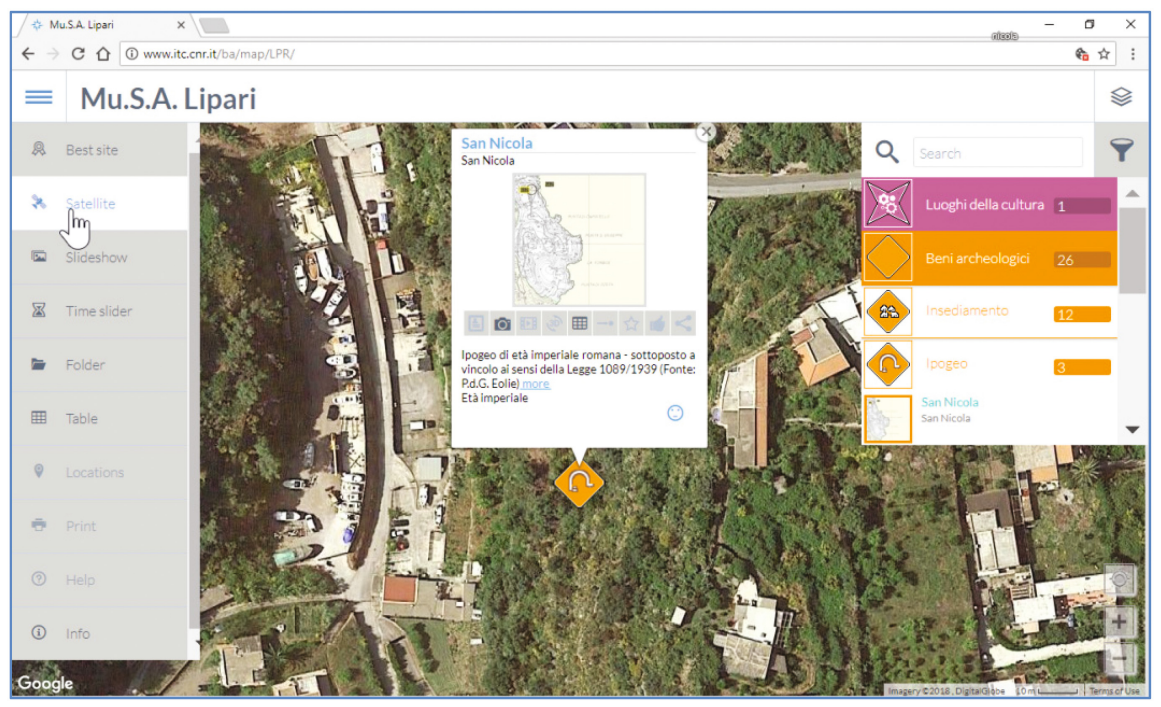

Fig. 10. Desktop map interface of Lipari map in 'earth view' (obtained clicking on 'Satellite' function in the menu). The three main components are opened: 'Menu' (left), 'Callout' (center), and 'Sidebar' (right). The first layer in the Sidebar has a grey color, therefore the P.o.I.s belongings to this category are not displayed in the map.

A command panel on bottom contains a 'Tiles on/off' icon aiming to display the previews all together in a scrolling window (Fig. 11).

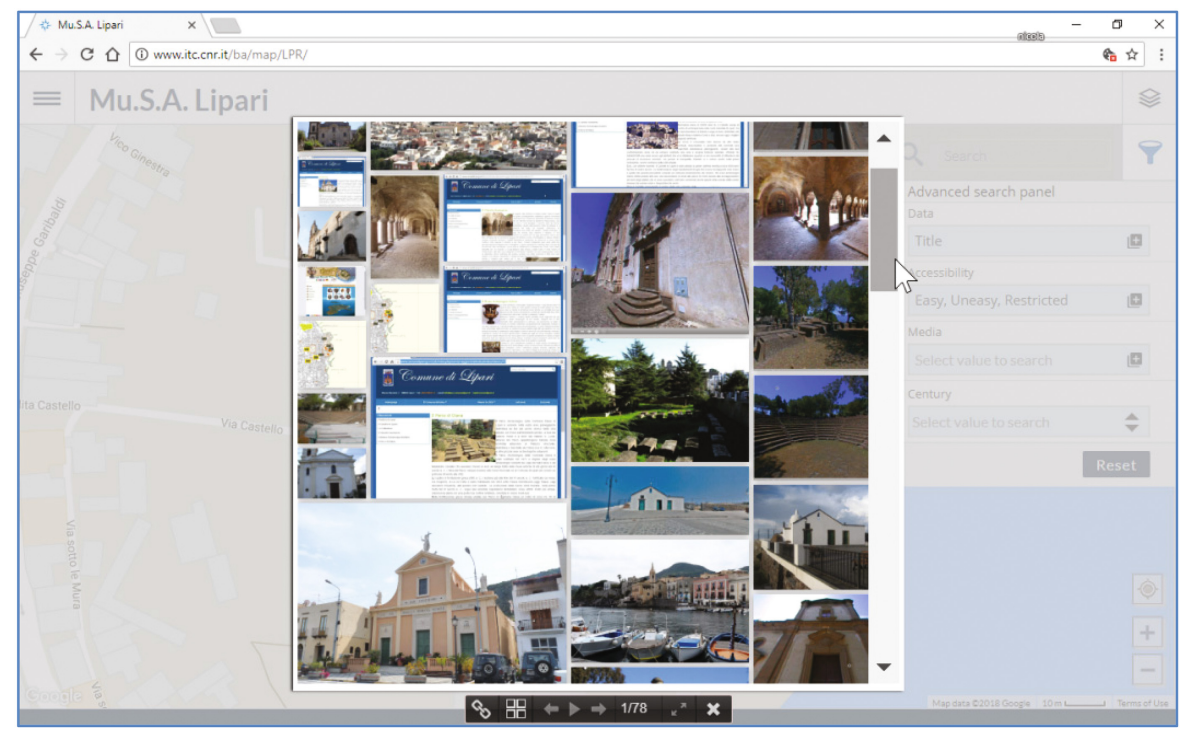

Fig. 11. Multimedia preview in a scrolling window ('Tile') opened in 'Folder' from 'Menu'. 
The user is allowed: (a) to locate the respective P.o.I., by clicking on the preview; (b) to view the multimedia, which the preview refers to-as the virtual tour for the Norman Cloister and the Diana's Park, available in the Internet, developed using only one 360-degree panorama (Fig. 12).
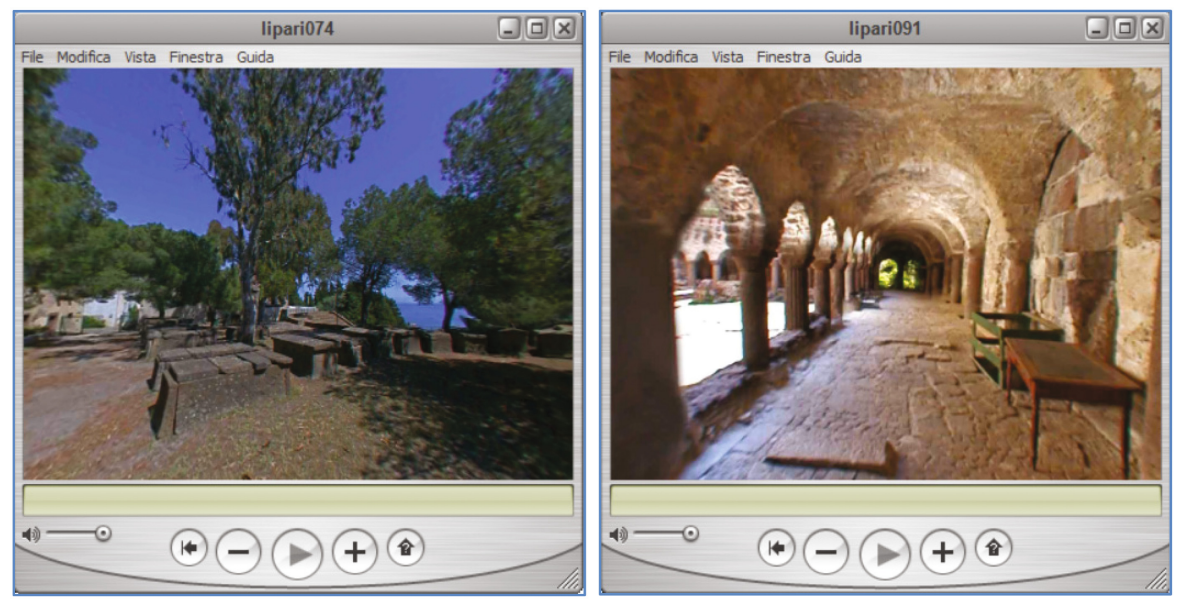

Fig. 12. Virtual tour for the Norman Cloister (left) and the Diana's Park (right), available in the Internet, developed using only one 360-degree panorama.

- 'Table', listing the media items, here belonging to all the sites in the project, using a panel with columns (Item, Site, Source, Date, Media Type, and Content) alphabetically ordered.

A 'Search' function allows finding multimedia according to the letters typed in the textbox. The user is allowed to see the multimedia content by clicking on an item.

Callout. This component is a window-opened by clicking on a P.o.I. box in the sidebar or on a P.o.I. marker on the map-showing:

- Two-line header (name and address).

- P.o.I. characteristic square image, used also in the slideshow and in the sidebar);

- Function icons, allowing user to have a preview of multimedia contents belonging to the selected P.o.I. ('text', 'image', 'video', and '3D') or their list ('Table')—other functions are in development.

- A short description (expandable clicking on 'more').

- The building age (used by the 'time slider' function).

- An accessibility status icon, showing three kind of image according to the physical accessibility of the P.o.I. - easy (accessible to all, at all times), uneasy or restricted. This kind of information has an additional cost because requests on-site survey, but it is fundamental. People using a wheelchair may have no difficulty at home in 
finding information about P.o.I.s but, when they get to their destination, they may find that they cannot reach it because of - for example - the presence of staircases at the entrance.

Sidebar. This component is a two-side window showing the symbols of search and layer. Clicking on the 'Sidebar' symbol the application shows/hides the first window (Fig. 10 on the right) having the search box, the filter symbol and the dynamic legend, organized in three levels:

- The first level depicts the main layers, using markers according to the P.o.I.s categories. If the category is divided in types, its marker in the 'Sidebar' has no images/text inside, as for 'Archeological Site' category.

- The second level depicts the sub-layers according to the P.o.I.s types (if any) for the selected category, as 'Village', 'Hypogeum', 'Wreck', and 'Remains' for 'Archeological Sites' category.

- The third level shows P.o.I. info boxes for the selected category (and type), displaying square picture, name, and address, as 'San Nicola' (Fig. 10).

Each box at the first and second level shows the number of the P.o.I. included, according to the search results and status of selections in the filter panel.

The user, according to its interest, could turn off/on each layer or each sub-layer, disabling/enabling the respective point's visualization, thus enhancing the map view.

Clicking on the 'Filter' symbol shows/hides the second window substituting the dynamic legend with the filter panel, allowing the user to set:

- 'Data for search' (Title, Address, and Comment—default: title).

- 'Accessibility level' (Easy, Uneasy, and Restricted—default: all).

- 'Multimedia type' (Sheet, Image, Video, and 3D — default: all).

- 'Building Century' (according to P.o.I. data-default: all).

\section{Conclusions}

This paper presents the design and implementation of a multimedia map loaded in a single HTML page having multiple features and functions, as distinct from other maps, to support users in searching for cultural heritage information according to their needs.

Future work will focus on the completion of functions provided in the callout ('Destination', 'Favorite', 'Like', and 'Share') for the desktop map interface and the improvement of the 'Language' tab in order to give users to load a whole set of labels corresponding to those utilized in the desktop map interface.

The multimedia demonstration map developed has the potential to help foster greater knowledge about, and resulting awareness of, the natural and cultural heritage objects.

Author Contributions. Maiellaro, N.: project and testing $(\S 1,4)$; Lerario, A.: case study $(\S 2)$; Varasano, A.: system architecture and software development (§3). 


\section{References}

1. Travel and Tourism Competitiveness Report 2015. http://www3.weforum.org/docs/TT15/ WEF_Global_Travel\&Tourism_Report_2015.pdf. Accessed 03 Mar 2018

2. Guggenheim, J., Kremser, S., Jhunjhunwala, P., McCaleb, T., Garcia-Mon, A., McCabe, L.: Travel goes mobile. http://img-stg.bcg.com/Travel_Goes_Mobile_Jun_2014_tcm9-84752. pdf. Accessed 03 Mar 2018

3. Peterson, M.P.: Hypermedia maps and the internet. In: Stefanakis, E., Peterson, M.P., Armenakis, C., Delis, V. (eds.) Geographic Hypermedia. Lecture Notes in Geoinformation and Cartography, pp. 121-136. Springer, Heidelberg (2006). https://doi.org/10.1007/978-3540-34238-0_7

4. Cartwright, W.E., Hunter, G.J.: Towards a methodology for the evaluation of multimedia geographical information products. GeoInformatica 5(3), 291-315 (2001)

5. Hu, S.: Multimedia mapping on the internet using commercial APIs. In: Peterson, M.P. (ed.) Online Maps with APIs and WebServices. Lecture Notes in Geoinformation and Cartography, pp. 61-71. Springer, Heidelberg (2012). https://doi.org/10.1007/978-3-64227485-5_5

6. Southern Illinois University Edwardsville - Maps \& Directions. http://www.siue.edu/maps/ index2.shtml. Accessed 05 Apr 2018

7. Cacia, C.: The activity of UNESCO regarding the element of recovery of the cultural identity: the example of Aeolian Islands. In: Claval, P., Pagnini, M.P., Scaini, M. (eds.) The Cultural Turn in Geography: Proceedings of the Conference, pp. 347-354, 18-20 September 2003, Gorizia Campus (2003)

8. Anzidei, M., et al.: Impact of the relative sea level rise on archaeological and cultural heritage sites of Lipari island (Italy), between the Roman period and 2100 AD. Geophysical Research Abstracts, vol. 20, EGU2018-12015-1, 2018 EGU General Assembly (2018)

9. Ruggieri, G., Calderon Vazquez, F.J.: Tourism development in UNESCO natural heritage sites - the case of sicilian volcanic sites: Mount Etna and Aeolian Islands. Chin. Bus. Rev. 16(11), 544-554 (2017). https://doi.org/10.17265/1537-1506/2017.11.002

10. Geremia, F., Massoli-Novelli, R.: Coastal geomorphosites of the isles of Lipari and Stromboli (Aeolian Islands, Italy): new potential for geo-tourism. Il Quaternario Italian J. Quat. Sci. 18(1), 231-242 (2005). Volume Speciale

11. Bui, H.T., Le, T.A., Nguyen, C.H.: UNESCO world heritage designation. In: Tourism and Opportunities for Economic Development in Asia, January 2017. https://doi.org/10.4018/ 978-1-5225-2078-8.ch015

12. Caust, J., Vecco, M.: Is UNESCO world heritage recognition a blessing or burden? Evidence from developing Asian countries. J. Cult. Herit. 27, 1-9 (2017). https://doi.org/10.1016/j. culher.2017.02.004

13. Di Matteo, D.: Tourism performances, government effectiveness and local growth. The Italian cultural heritage perspective. In: Gursoy, D., Nunkoo, R. (eds.) Routledge Handbook of Tourism Impacts: A Theoretical Perspective. Routledge, London (2019)

14. Patuelli, R., Mussoni, M., Candela, G.: The effects of world heritage sites on domestic tourism: a spatial interaction model for Italy. J. Geograph. Syst. 15, 369-402 (2013)

15. Lo Piccolo, F., Leone, D., Pizzuto, P.: The (controversial) role of the UNESCO WHL management plans in promoting sustainable tourism development. J. Policy Res. Tour. Leisure Events 4(3), 249-276 (2012). Tourism and Spatial Planning

16. VanBarclom, B.L.: Assessing the economic impact of a UNESCO World Heritage designation. J. Herit. Tour. 6(2), 143-164 (2011) 
17. Cuccia, T., Rizzo, I.: Seasonal tourism flows in UNESCO sites: the case of Sicily. In: Kaminski, J., Benson, A.M., Arnold, D. (eds.) Contemporary Issues in Cultural Heritage Tourism, pp. 179-199. Routledge, London (2014)

18. Cellini, R., Cuccia, T.: UNESCO sites as public goods: comparative experiences in Italy. Revista de Economia Contemporânea 20(3), 553-569 (2016)

19. Places Mirabilia Network of Unesco Sites. http://www.mirabilianetwork.eu/en/placesmirabilia-network-of-unesco-sites. Accessed 05 Apr 2018

20. Ministry of Heritage and Cultural Activities and Tourism of Italy. www.catalogo. beniculturali.it/sigecSSU_FE/mappaRegione.action?regione=19\&r=sicilia\&T= 1521820871495. Accessed 03 Mar 2018

21. Department of Cultural Heritage and Sicilian Identity. http://www.regione.sicilia.it/ beniculturali/dirbenicult/database/museogeo/Site_Locator.asp?zoom=8. Accessed 03 Mar 2018

22. Official website of Lipari. http://www.comunelipari.gov.it/lipari/zf/index.php/serviziaggiuntivi/index/index/idtesto/73. Accessed 03 Mar 2018

23. Aeolian Islands UNESCO Management Plan - Cartographic attachments. http:// unescosicilia.it/wp/wp-content/uploads/2014/09/2-Allegati-Cartografici.pdf. Accessed 03 Mar 2018

24. What is an Entity Relationship Diagram - Entity-relationship model. https://www.lucidchart. com/pages/er-diagrams. Accessed 04 Apr 2018

25. Proceedings of the combined volumes of International Congress (IntCongress 2014) held at Holiday Inn Silom, Bangkok, Kingdom of Thailand between 19th November, 2014 and 21st November, 2014

26. Datatable - A plug-in for the jQuery Javascript library. https://datatables.net/. Accessed 04 Apr 2018

27. Scott Jr., E.A.: SPA Design and Architecture: The Communication Process, November 2015. ISBN 9781617292439. http://freecontent.manning.com/wp-content/uploads/spadesign-and-architecture-the-communication-process.pdf. Accessed 04 Apr 2018

28. Single-page-application Vs multiple-page-application. https://medium.com/@NeotericEU/ single-page-application-vs-multiple-page-application-2591588efe58. Accessed 04 Apr 2018

29. RESTful Service Best Practices. http://www.restapitutorial.com/media/RESTful_Best_ Practices-v1_1.pdf. Accessed 04 Apr 2018

30. AJAX, Asynchronous JavaScript And XML. https://developer.mozilla.org/en-US/docs/Web/ Guide/AJAX. Accessed 13 May 2018

31. Hatami, S.: The Automatic Development of SEO-Friendly Single Page Applications Based on HIJAX Approach, October 2017. https://doi.org/10.1007/978-3-319-68786-5_15, https:// www.researchgate.net/publication/320184470_The_Automatic_Development_of_SEOFriendly_Single_Page_Applications_Based_on_HIJAX_Approach. Accessed 13 May 2018

32. Google Search console, Googlebot crawler support. https://support.google.com/webmasters/ answer/6066468. Accessed 13 May 2018

33. Schwartz, B.: Google will stop using the old AJAX crawling scheme in Q2 2018. Search Engine Land, December 2017. https://searchengineland.com/google-will-stop-crawling-oldajax-crawling-scheme-q2-2018-287653. Accessed 13 May 2018

34. Jenny, B., Kelso, N.V.: Color Design for the Color Vision Impaired in Mapping: Methods Tips. Cartographic perspectives, no. 57, pp. 61-67, Spring 2007. http://colororacle.org/ resources/2007_JennyKelso_ColorDesign_hires.pdf. Accessed 03 Mar 2018 
Open Access This chapter is licensed under the terms of the Creative Commons Attribution 4.0 International License (http://creativecommons.org/licenses/by/4.0/), which permits use, sharing, adaptation, distribution and reproduction in any medium or format, as long as you give appropriate credit to the original author(s) and the source, provide a link to the Creative Commons licence and indicate if changes were made.

The images or other third party material in this chapter are included in the chapter's Creative Commons licence, unless indicated otherwise in a credit line to the material. If material is not included in the chapter's Creative Commons licence and your intended use is not permitted by statutory regulation or exceeds the permitted use, you will need to obtain permission directly from the copyright holder.

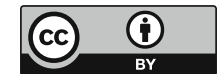

